\title{
Measurements of jet quenching using the ATLAS detector
}

\section{Martin SPOUSTA* for the ATLAS collaboration}

Charles University in Prague, Faculty of Mathematics and Physics

E-mail: spousta@ipnp.troja.mff.cuni.cz

We present a study of the performance of jet reconstruction algorithms for $\mathrm{Pb}+\mathrm{Pb}$ collisions at $5.52 \mathrm{TeV} / \mathrm{n}$ based on a full GEANT simulation of the ATLAS detector. We show that ATLAS can provide measurements that are sensitive to different in-medium energy loss scenarios leading to the jet quenching. We present the capability of measurements of fragmentation function and jet shapes. Sensitivity of these measurements to a possible modification by the jet quenching is discussed. We also briefly discuss the capabilities to measure di-jet and $\gamma$-jet correlations as well as jet $R_{\mathrm{AA}}$.

High-pT Physics at LHC -09

February 4- 42009

Prague, Czech Republic

\footnotetext{
* Speaker.
} 
Measurements of jets and their properties provide information about the jet quenching phenomenon that is predicted for heavy ion collisions and already measured at RHIC. The jet quenching means that a hard parton, before fragmenting into a jet of hadrons, deposits a fraction of its energy in the medium, leading to a change of jet energy distribution and particle multiplicity inside a jet. At RHIC experiments the jet quenching phenomenon has been clearly established in the measurement of single- and di-hadron spectra [1]. Due to the limited $p_{\mathrm{T}}$ reach of these measurements and the large soft background, they suffer from biases towards particles that escape the medium having lost little energy. In contrast, the high center-of-mass energy and large rates for high- $p_{\mathrm{T}}$ jets at the LHC will make full jet measurements possible over a wide range of jet energies. In these proceedings we present capabilities of the ATLAS detector [2] for measurements of jets and their properties in heavy ion collisions. In the first section we briefly describe the jet reconstruction strategy. In the second section we show the basic performance of the cone jet reconstruction. In the third section we discuss measurements of variables sensitive to the radiative energy loss. We also show the expected effect of jet quenching to be observed in these measurements. In the last section we discuss measurements sensitive to the collisional energy loss.

\section{Jet reconstruction strategy}

Twenty million jets with $E_{\mathrm{T}}>50 \mathrm{GeV}$ is expected to be produced during one run of $\mathrm{Pb}+\mathrm{Pb}$ collisions at LHC [3]. These high rates of the jet production will be accompanied by the large background from the underlying heavy ion event. Thus, it is important to use a suitable strategy to deal with the underlying event during the reconstruction. We have developed and tested two strategies, one based on the iterative seeded cone algorithm and the other based on the fast $k_{\mathrm{T}}$ algorithm [4]. In these proceedings we concentrate on the results obtained from the cone algorithm reconstruction. Results and discussion of the fast $k_{\mathrm{T}}$ algorithm can be found in [5]. The strategy based on the cone algorithm is the following: First the average background is estimated as a function of pseudorapidity and calorimeter segmentation. In order not to overestimate the background by a possible presence of a jet, the background is estimated outside of the jet candidate regions that are found using a sliding window algorithm. After the subtraction of the background the standard cone jet algorithm and standard proton-proton calibrations are applied, using the cone size, $R=\sqrt{\Delta \eta^{2}+\Delta \phi^{2}}=0.4$.

\section{Basic performance results}

In order to evaluate the performance of the heavy ion jet reconstruction we used full reconstruction of PYTHIA [6] di-jet events embedded into simulated Pb+Pb HIJING [7] events. HIJING events were simulated without quenching and without hard scattering cut which would remove mini-jets. The analysis was carried out for three centrality selections: $b=2,6$, and $10 \mathrm{fm}$ which correspond to the charged particle multiplicity $\mathrm{d} N / \mathrm{d} \eta=2700,1700$ and 460 respectively. The performance results have been evaluated as a function of background multiplicity. The left plot of Fig. 1 shows the jet energy resolution as a function of the truth jet energy and as a function of centrality. It is visible that even in central collisions $(b=2 \mathrm{fm}, \mathrm{d} N / \mathrm{d} \eta=2700)$ the jet energy resolution is better than $25 \%$ for jets with energy above $80 \mathrm{GeV}$. Jet energy resolution improves 

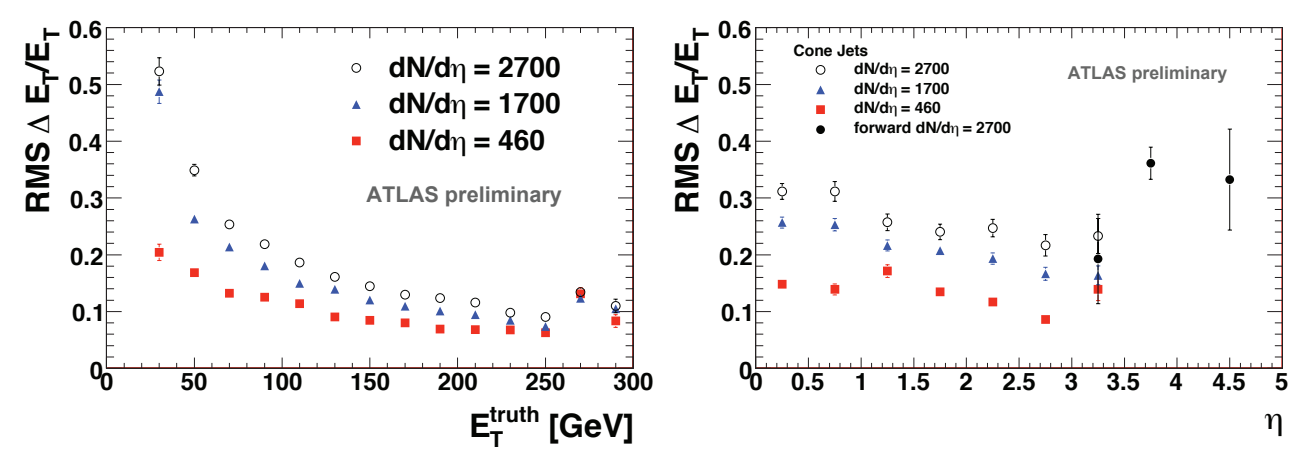

Figure 1: Left: Jet energy resolution as a function of the truth jet energy for different centrality bins. Right: Jet energy resolution as a function of pseudorapidity for different centrality bins.

with decreasing centrality. The right plot of Fig. 1 shows the jet energy resolution as a function of pseudorapidity. The analysis of the jet energy resolution for forward jets with pseudorapidity $|\eta|>3.2$ has only been performed for central collisions. In the forward pseudorapidity regions, two opposite effects contribute to the energy resolution: the calorimeter segmentation is not as fine as in the midrapidity region, but the underlying background is lower than at midrapidity. In consequence, the jet energy resolution in the forward pseudorapidity region is comparable to that at midrapidity. In summary, over the entire $\eta$ coverage of the calorimeter, the energy resolution stays around $20-30 \%$ in the highest multiplicity $\mathrm{Pb}+\mathrm{Pb}$ collisions.

The left plot of Fig. 2 shows the jet reconstruction efficiency as a function of the truth jet energy. It can be seen that there is little or no dependence of the efficiency on the underlying event multiplicity. The shape of the efficiency curve is influenced by the $5 \mathrm{GeV}$ tower $E_{\mathrm{T}}$ seed cut in the cone algorithm. Higher seed cuts would increase the efficiency, but would also increase the fake rate. The fake rate is presented in the right plot of Fig. 2, showing the jet $E_{\mathrm{T}}$ spectra. The reconstructed spectrum matches the truth spectrum quite well above $80 \mathrm{GeV}$. It was possible to improve the reconstructed jet $E_{\mathrm{T}}$ spectrum by applying a special procedure to eliminate fake jets. The rejection was done by making a cut on the variable sensitive to the shape of the energy distribution within the jet. More details can be found in $[8,9]$. In order to reach the desired efficiency and purity, the optimal choice of the variables with tuned cuts needs to be worked out as a function of the background characteristics. It is worth to note that the actual background level is likely to be substantially lower than the underlying event produced by HIJING without quenching.

\section{Fragmentation function and jet shapes}

Hard-scattered partons are expected to lose their energy via gluon bremsstrahlung in the hot and dense QCD medium. This gluon radiation leads to a modification of the jet energy flow and particle multiplicity in a jet. One of the measures sensitive to the radiative energy loss is the fragmentation function [10], $D(z)=1 / N_{\text {jet }} \mathrm{d} N / \mathrm{d} z$. For jets $z$ is the longitudinal momentum fraction of a jet fragment with respect to the jet axis. The fragmentation function is built up from charged particle tracks, extrapolated from the inner detector to the calorimeter, that match the reconstructed jet. The left plot of Fig. 3 shows the fragmentation function reconstructed in central collisions, 

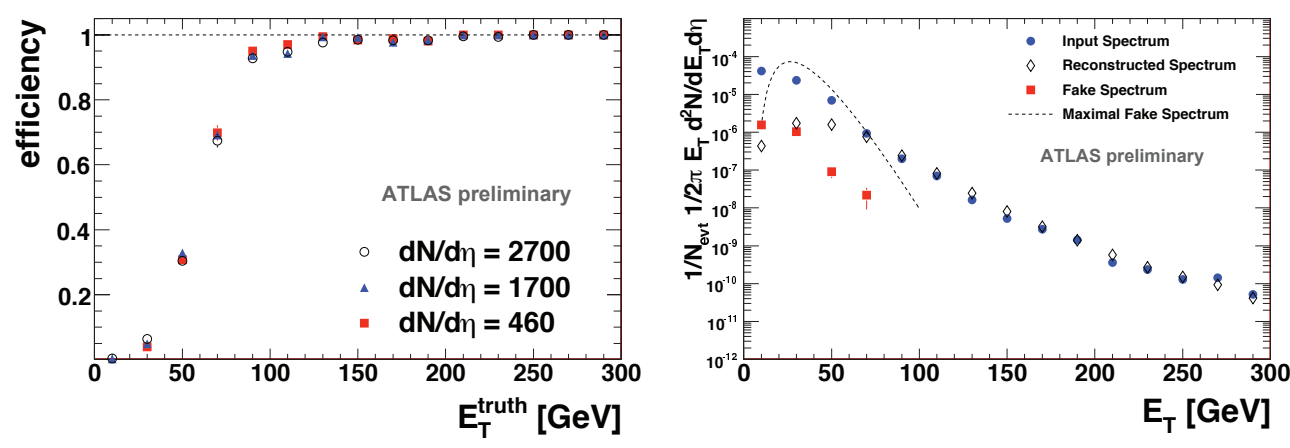

Figure 2: Left: Jet efficiency as a function of the truth jet energy for different centrality bins. Right: Input (filled circles), raw reconstructed (diamonds), and fake (squares) spectra for cone jets reconstructed in central collisions $(b=2 \mathrm{fm}, \mathrm{d} N / \mathrm{d} \eta=2700)$.

compared to the truth fragmentation function computed using charged particles from PYTHIA. The $p_{\mathrm{T}}$ cut used for charged particle tracks was $p_{\mathrm{T}}>2 \mathrm{GeV}$. The jet energy is in the range from 70 to $140 \mathrm{GeV}$. There are several corrections that needed to be applied to obtain such a good agreement between the truth and reconstructed distributions: tracking efficiency correction, jet position resolution correction and subtraction of the background distributions. Detailed description of these corrections can be found in [11]. To test the sensitivity to possible modifications of the fragmentation function by the dense QCD medium, the PYQUEN event generator [12] was used which includes the jet quenching effects. The right plot of Fig. 3 shows the difference between reconstructed fragmentation functions from PYTHIA and PYQUEN. Similarly to the result presented in [10], we can see that the high- $z$ fragments are suppressed and the low- $z$ fragments are enhanced. It is clear that expected in-medium modification of the fragmentation function is well within the ATLAS measurement capability.

The ATLAS detector is well suited for calorimetric measurements of the energy flow inside the jet, thus the jet shape. The integral jet shape measures the energy flow at different distances $r$ from the jet axis, $\Psi\left(r, R_{\text {cone }}\right)=\int_{0}^{r} E_{\mathrm{T}}(\rho) \mathrm{d} \rho / \int_{0}^{R_{\text {cone }}} E_{\mathrm{T}}(\rho) \mathrm{d} \rho$. In addition to the integral jet shape we can study the differential jet shape $\psi\left(r, R_{\text {cone }}\right)$ which is a derivative of the integral jet shape. The left plot in Fig. 4 shows the comparison between differential jet shapes reconstructed in central collisions ( $b=2 \mathrm{fm}, \mathrm{d} N / \mathrm{d} \eta=2700)$ and in peripheral collisions $(b=10 \mathrm{fm}, \mathrm{d} N / \mathrm{d} \eta=460)$. It is visible that, after the background subtraction and application of the jet position resolution correction, we can well reproduce jet shapes even in central collisions. Similarly to the fragmentation function it is expected that also jet shapes will be modified by the presence of the dense QCD medium [13]. To test the sensitivity to possible modifications of jet shapes by the medium we have used PYQUEN event generator. The right plot of Fig. 4 shows the difference between the reconstructed differential jet shapes from PYTHIA and PYQUEN. Quite sizable differences are visible, similar to those reported in [13]. The energy is redistributed from the center of the jet to the jet periphery.

Sensitivity of the jet shape and fragmentation function needs to be evaluated also in the presence of the heavy ion background which has not been done yet. The results shown in Fig. 3 (left) and Fig. 4 (left) suggest that the effect of the background can be well handled and that, in consequence, the ATLAS is capable to measure modifications of jets by the radiative energy loss. 

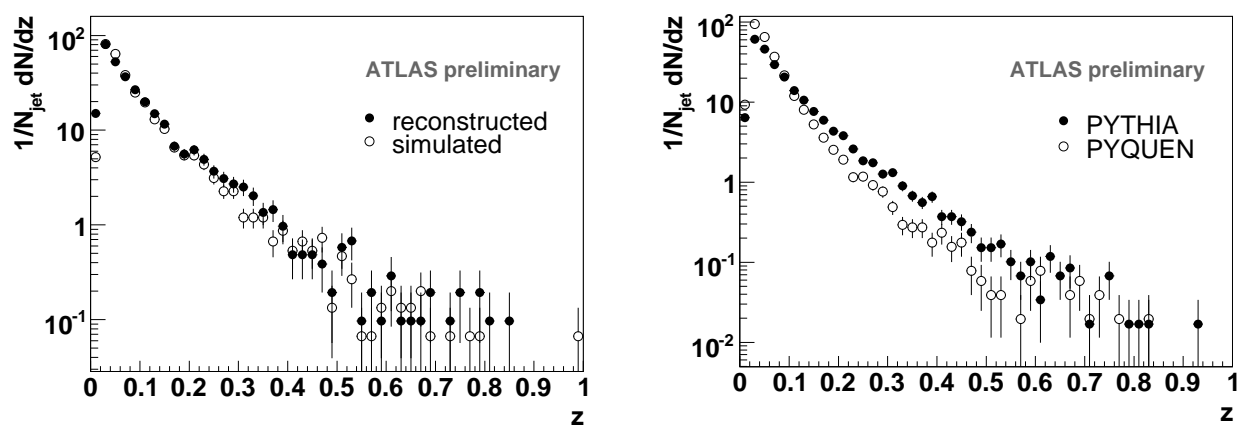

Figure 3: Left: Fragmentation function for jets in the central collisions after all corrections and background subtraction (full circles). The truth fragmentation function is shown by open circles. Right: Fragmentation function for reconstructed PYTHIA (open) and PYQUEN (full) events.
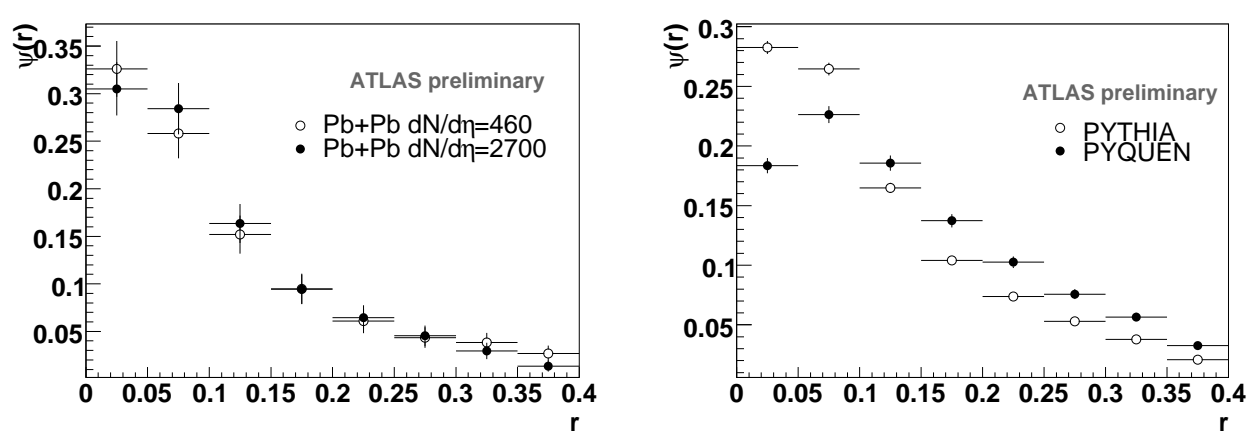

Figure 4: Left: Differential jet shape for jets in central (full) and peripheral (open) collisions after all corrections and background subtraction. Right: Differential jet shape from reconstructed PYTHIA (open) and PYQUEN (full) events.

\section{Correlation measurements and jet $R_{\mathrm{AA}}$}

The large $\eta$ acceptance of the ATLAS calorimeter system provides nearly complete acceptance for di-jets making possible correlation measurements between back-to-back jets. Except the radiative energy loss which can be measured using variables described in the previous section, collisional energy loss may also play a significant role in the parton energy loss [14]. In the case of the collisional energy loss the energy is transferred out of the jet cone due to multiple elastic scatterings of a parton with the constituents of the medium. Thus, such energy loss can be measured via the modification of the di-jet spectra. The left plot of Fig. 5 shows the conditional yield for detecting an associated jet above $70 \mathrm{GeV}$ given the leading jet above $100 \mathrm{GeV}$, plotted as a function of $|\Delta \phi|$ between the two reconstructed jets. Integrating the distribution over $|\Delta \phi|$ gives high, $60 \%$ probability for detecting an associated jet. Another useful tool to study the jet energy loss are the measurements of $\gamma$-jet correlations. Since photons do not interact strongly, they can be used to estimate the original energy and direction of a jet interacting with the medium. Measurements of $\gamma$ jet correlation can benefit from excellent longitudinal segmentation of the calorimeter $(|\Delta \eta|=0.003$ 

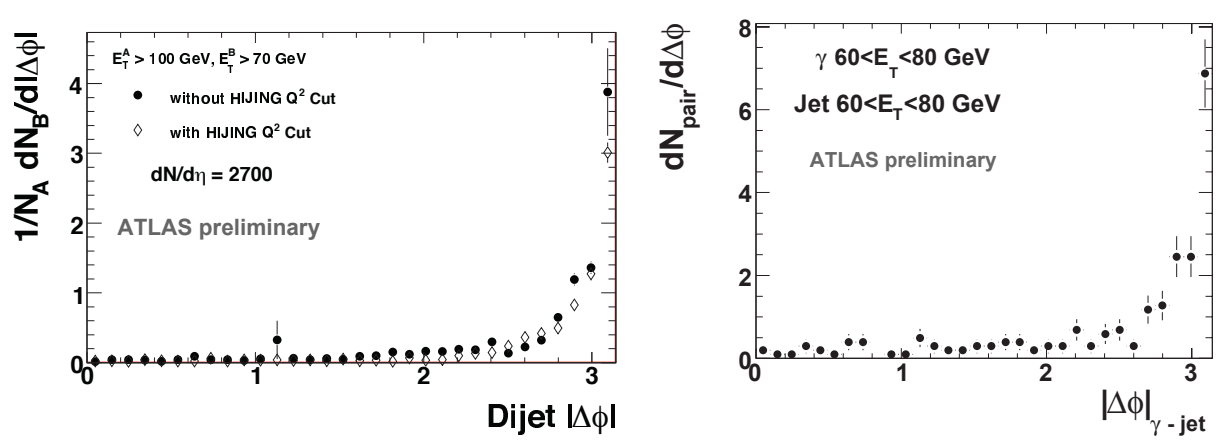

Figure 5: Left: Conditional yield for detecting an associated jet with $E_{\mathrm{T}}$ above $70 \mathrm{GeV}$ given the leading jet with $E_{\mathrm{T}}$ above $100 \mathrm{GeV}$. PYTHIA jets were embedded in HIJING events with (open) and without (full) hard scattering cut used to remove mini-jets. Right: Conditional yield for detecting an associated $\gamma$ with $E_{\mathrm{T}}$ between 60 and $80 \mathrm{GeV}$ given a jet with the same energy in the event.
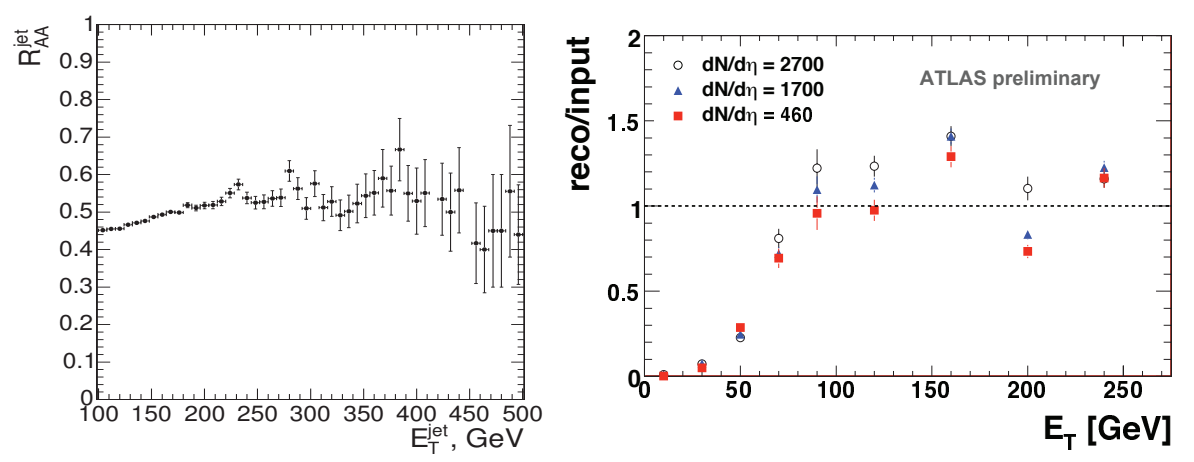

Figure 6: Left: Expected jet $R_{\mathrm{AA}}$ from Ref. [14]. Right: Reconstructed to input jet spectra without any correction as a function of the truth jet energy.

for the first sampling of the electromagnetic calorimeter). This fine segmentation allows for a good separation between the direct and background photons [15, 5]. The right plot of Fig. 5 shows the conditional yield for detecting an associated $\gamma$ with $E_{\mathrm{T}}$ between 60 and $80 \mathrm{GeV}$ given a jet with the same energy in the event. It can be seen that the correlation measurements can be done with a good accuracy.

Another useful variable that could uncover the features of collisional energy loss is a measurement of the jet $R_{\mathrm{AA}}$. The left plot in Fig. 6 shows the expected modification of the jet $R_{\mathrm{AA}}$ predicted by the PYQUEN model [14]. The size of the modification is of the order of 50\%. This can be compared with the precision of the jet rate measurements. The right plot of Fig. 6 shows the ratio of the reconstructed to input spectra without any corrections applied to the reconstructed spectrum. One can see that the achieved precision is better than $20 \%$ for jets with $E_{\mathrm{T}}>80 \mathrm{GeV}$. Such precision is sufficient to measure the expected modification from the collisional energy loss. For more details see also Refs. [16, 5]. 


\section{Conclusions}

Utilizing the large acceptance, finely-segmented ALTAS calorimeter, jets in heavy ion environment can be measured with high efficiency and excellent energy resolution over a broad range in energy and pseudorapidity. ATLAS can provide a set of measurements that are sensitive to different energy loss mechanisms. Thus, we expect that together with other LHC experiments ATLAS will shed a new light on the mechanisms of the parton energy loss in the hot and dense QCD medium.

This work was supported in part by the grant of Ministry of Education, Youth and Sports, Czech Republic LA 08032, LC 527.

\section{References}

[1] M. van Leeuwen, J. Phys. G: Nucl. Part. Phys. 34 (2007) S559-S566

[2] The ATLAS Collaboration, G. Add et al., JINST 3 (2008) S08003

[3] A. Accardi, N. Armesto, and I.P.Lokhtin, hep-ph/0211314 (2002)

[4] M. Cacciari, hep-ph/0607071

[5] N. Grau for the ATLAS Collaboration, J. Phys. G: Nucl. Part. Phys. 35 (2008) 104040

[6] T. Sjostrand, S. Mrenna and P. Skands, JHEP 0605 (2006) 026

[7] M. Gyulassy and X.-N. Wang, Comput. Phys. Commun. 83 (1984) 307

[8] N. Grau, B.A. Cole, W.G. Holzmann, M. Spousta and P. Steinberg for the ATLAS Collaboration, arXiv:0810.1219

[9] N. Grau, this conference proceedings

[10] C.A. Salgado, U.A. Wiedemann, Phys. Rev. Lett. 93 (2004) 042301

[11] M. Spousta for the ATLAS Collaboration, ATL-PHYS-PROC-2009-002

[12] I.P. Lokhtin, A.M. Snigirev, hep-ph/0406038

[13] I. Vitev, S. Wicks, B.W. Zhang, JHEP 11 (2008) 093

[14] I.P. Lokhtin, S.V. Petrushanko, A.M. Snigirev, C.Yu. Teplov, arXiv:0706.0665v1

[15] J. Jia for the ATLAS Collaboration, ATL-PHYS-PROC-2008-047

[16] M. Spousta for the ATLAS Collaboration, ATL-PHYS-PROC-2009-022 